A response surface analysis investigation of the effects of (mis)alignment between interpersonal values and efficacies on interpersonal problems

\author{
by
}

Madeline Kehl

Submitted to the Graduate Faculty of

University of Pittsburgh in partial fulfillment

of the requirements for the degree of

Bachelors of Philosophy

University of Pittsburgh

2019 


\section{UNIVERSITY OF PITTSBURGH}

Dietrich School of Arts and Sciences

This thesis was presented

by

\section{Madeline Kehl}

It was defended on

November 8, 2019

and approved by

Dr. Michael Pogue-Geile, Clinical Program Chair, Psychology

Dr. Joseph Beeney, Assistant Professor, Psychiatry

Dr. Chris Hopwood, Associate Professor, Psychology

Advisor/Dissertation Director: Dr. Aidan G.C. Wright, Associate Professor, Psychology 
Copyright (C) by Madeline Kehl

2019 


\title{
A response surface analysis investigation of the effects of (mis)alignment between interpersonal values and efficacies on interpersonal problems
}

\author{
Madeline Kehl \\ University of Pittsburgh, 2019
}

This paper used response surface analysis (RSA) to examine the relationship between various alignments and misalignments in interpersonal values and efficacies and interpersonal problems. Based on previous research and theory, we expected discrepancies between these personality levels to be associated with interpersonal problems, as internal conflict has generally been linked to distress. In addition, as interpersonal values can be taken to represent participants' ideal interpersonal states, and interpersonal efficacies, participants' perceived achievable interpersonal states, a mismatch between them may be associated with negative outcomes as they parallel the concept of a goal and perceived ability to reach the goal.We found that in general, misalignment was associated with increased interpersonal problems. More specifically we found that within an interpersonal dimension (such as warmth) having an efficacies greater than values mismatch was associated problems with the low pole of that dimension (in this case, coldness). These results anchor the maladaptivity of internal conflict into a broader personality theory. 
$\begin{array}{ll}1.0 \text { Introduction } & 1\end{array}$

$\begin{array}{ll}\text { 2.0 Methods } & 7\end{array}$

$\begin{array}{ll}\text { 2.1 Participants } & 7\end{array}$

$\begin{array}{ll}\text { 2.1.1 Undergraduate sample } & 7\end{array}$

$\begin{array}{lr}\text { 2.1.2 Community sample } & 8\end{array}$

$\begin{array}{ll}\text { 2.2 Materials } & 8\end{array}$

$\begin{array}{ll}\text { 2.2.1 Circumplex Scales of Interpersonal Values } & 8\end{array}$

$\begin{array}{ll}\text { 2.2.2 Circumplex Scales of Interpersonal Efficacies } & 8\end{array}$

2.2.3 Inventory of Interpersonal Problems 9

2.3 Data Analytic Plan 9

$\begin{array}{ll}\text { 3.0 Results } & 12\end{array}$

$\begin{array}{lr}\text { 3.1 Primary analyses } & 12\end{array}$

3.1.1 Effects with quantitative and interpretive replication $\quad 12$

3.1.2 Effects with quantitative but not interpretive replication $\quad 13$

3.1.3 Effects with interpretive but not quantitative replication $\quad 13$

3.1.4 Effects that did not replicate $\quad 14$

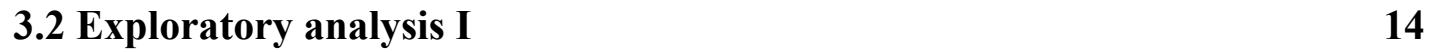

3.2.1. Effects with quantitative and interpretive replication $\quad 14$

3.2.2 Effects with quantitative but not interpretive replication $\quad 15$

3.2.3 Effects with interpretive but not quantitative replication $\quad 15$ 
3.3.1. Effects with quantitative and interpretive replication

4.0 Discussion 


\section{List of Figures}

$\begin{array}{ll}\text { Figure } 1 \text { Interpersonal Circumplex } & 25\end{array}$

$\begin{array}{ll}\text { Figure } 2 \text { Results, Primary Analysis } & 26\end{array}$

$\begin{array}{ll}\text { Figure } 3 \text { Results, Exploratory Analysis I } & 27\end{array}$

$\begin{array}{ll}\text { Figure } 4 \text { Results, Exploratory Analysis II } & 28\end{array}$ 


\subsection{Introduction}

Although interpersonal theory originated as a behavior-oriented response to Freudian emphasis on intrapsychic phenomena, due to the far-reaching implications of interpersonal experience and functioning, contemporary interpersonal theory has found relevance in diverse areas of inquiry, including the study of personality development and psychopathology (Pincus \& Ansell, 2012). As defined by Pincus and Ansell (2003) "interpersonal situation" refers to "a set of fundamental phenomena important for personality development, structuralization, and pathology...It is not meant to generate a dichotomy between what is inside the person and what is outside the person." Instead the 'interpersonal situation' describes the feedback loop of interpersonal experience and personality development created by evolving mental representations of self and other (Lukowitsky \& Pincus, 2011; Baldwin 1992).

In this way, contemporary interpersonal theory anchors a parallel processing model similar to Mischel and Shoda's (1998) cognitive-affective processing system (CAPS) model in the interpersonal situation. Both models reconcile trait stability with behavioral variability in individuals by framing personality as a flexible series of parallel processes with the salience and importance of each process subject to fluctuation across situations and between individuals (Fournier, Moskowitz \& Zuroff, 2008; Mischel \& Shoda, 1998). The “process” aspect of this definition implies variance of personality over time, while the "parallel" aspect indicates coexistence of multiple levels within a single personality system. This provides two avenues, temporal and structural, through which the overarching concept of personality dynamics can be described and investigated. These two aspects are, in a sense, mutually informative, with levels of personality structure able to be inferred from changes over time and changes over time 
requiring insight into personality structure to fully understand. However, ultimately for temporal variation to be legible the relationships between levels of personality must be definite. (Pincus et al 2014)

When Timothy Leary originally proposed personality structure as being comprised of levels, his were comprised of elements such as private symbolization (Level III) which post technical challenges to operationalize in a way that would support quantitative comparison both across people and to other levels within a person (Leary, 1957). Tory Higgins' self-discrepancy theory points to a more tractable set of intrapersonal levels. This work investigates the discrepancies between mental representations of actual/ideal self and actual/ideal perception of others' expectations and their impact upon psychological well being. Results stemming from this theory suggest that different misalignments produce different negative outcomes, with one's ideal surpassing one's actual self producing feelings of emptiness and with one's actual self and the perception of what others expect producing feelings of agitation (Higgins, 1987; Higgins, 1988).

Higgins' self-discrepancy theory aligns closely with the type of within-person dynamics proposed by Leary. At the same time, findings from self-discrepancy theory would be strengthened if they were tethered to an established personality model, rather than requiring new and possibly idiosyncratic constructs. Interpersonal theory provides a well-validated structural model of personality, the interpersonal circumplex (IPC) to organize questions related to misalignment in levels of personality and their outcomes. The IPC is a graphical representation of a matrix within which social-transactional and interaction-based concepts have been refined into a continuous array of variables, with the last and first correlating roughly as closely as the 
first and second (Leary, 1957; Wiggins, 1979; Conte, 1981; Kiesler, 1983; Foa 1961). This circumplex structure collapses trigonometrically onto the axes of dominance/submissiveness and warmth/coldness, that can then be used to describe a person's relative standing across these two orthogonal dimensions (see Figure 1). Furthermore, these axes, dominance and warmth, have served as a model of both adaptive and maladaptive personality development (Luyten \& Blatt, 2013; Hopwood, Pincus, \& Wright, 2019). For example, the circumplex has successfully been used to map and distinguish various DSM-labelled pathologies, including the DSM IV's "Axis I" and "Axis II" (Wright et al., 2012; Eldredge, Locke, Horowitz, 1998; Pincus, 2014). Although this demonstrates the utility of these axes for organizing essential information related to personality functioning, a one-dimensional, static measure, like that employed in the aforementioned research, does little to elucidate maladaptive versus adaptive personality dynamics. However the IPC has also been described as a "nomological net", forming a space that allows for the accurate measurement of many key manifestations of interpersonal functioning (Gurtman, 1992). The IPC can therefore become a metric not just for comparing individuals but for examining the alignment (or lack thereof) of processes or levels of personality within individuals.

Over the last few decades the measures developed for the IPC have set the scene to examine intrapersonal processes and conflicts occuring in the interpersonal sphere using a more comprehensive quantitative investigation. In particular, a variety of measures that capture different levels of interpersonal functioning have been developed that all use the IPC as their guiding framework, such as the Inventory of Interpersonal Problems (IIP; Alden, Wiggins, \& Pincus, 1990)) and the Circumplex Scale of Interpersonal Efficacies (CSIE; Locke \& Sadler, 
2007). Placing different aspects of functioning into the same structural space (the IPC) allows for direct mathematical comparison, thereby facilitating the analysis of level alignment within an individual across different levels of interpersonal functioning. This also anchors the question of internal conflict between levels in a rich theoretical space, an aspect which prior literature has lacked. For instance, the IPC's ability to answer similar questions has been exploited in previous work, primarily as a clinical tool to aid in helping a single individual identify internal inconsistencies and conflict across a large array of IPC measures (Pincus et al., 2014). By shifting the scope of investigation from an individual as a multi-level system to the relationship between specific levels of personality in the population, the IPC framework can provide insight into generalizable personality processes.

Two such areas of interpersonal functioning that can be captured using an IPC framework are values and efficacy. These constructs might be especially useful for understanding interpersonal distress given that, together, they capture one's goal and one's perceived ability to attain that goal. This is conceptually similar to Higgins's comparison of ideal self and actual self, however the interpersonal values and efficacies questionnaires also tap into broader literatures, like those of social-cognitive theory and attitudinal-behavioral theory. For example, there are several theoretical reasons to expect a person's values and efficacies to align. In many cases, an action's ability to produce results that people value motivates them to repeat that behavior in similar situations in the future (Bandura, 1977; Feather, 1982; Baldwin, 1992; Horowitz Rosenberg \& Bartholomew, 1993). It follows that if a person repeats a behavior with success, they are more likely to feel competent with regards to its execution. Indeed, a person's 
perception of their ability to act in accordance with an interpersonal value, is generally predictive of them reporting having that value (Locke \& Sadler, 2007).

However, there is reason to believe that a number of individuals may have mismatches in their values and efficacy. For example, while a combination of dominance and warmth in interpersonal style is strongly associated with well-being, at least in Western cultures, they are neither universal individual traits nor values. Part of this may be because, while being respected and accepted both generally appeal to humans, both incur the risk of failure, a nearly universally negative experience (Locke, 2015). How people evaluate the risk of acting in a way that could lead to rejection or ridicule and how they weigh those factors against potential positive outcomes may interfere with their decision to attempt behaviors they value. This prediction is in line with attitude-behavior theory; for example, a person may value close relationships or praise (positive attitude towards target) but feel negatively about expressing warmth or satisfaction due to fear of rejection or exploitation (negative attitude towards action) resulting in a reduced correlation between values and behavior (Azjen \& Fishbein, 1977). In other words, they doubt their ability to successfully enact the behavior most likely to obtain the optimal result, instead settling for a behavior with an acceptable result that they know they can achieve. This kind of disharmony might contribute to inconsistency in behavior, inappropriate responses, or responses that do not elicit satisfactory counter-responses from the other person (Kiesler, 1983). Any of the above would be indicative of psychopathology (Hopwood, Pincus \& Wright, 2019). By examining the alignment of interpersonal values and efficacies in the context of interpersonal problems, we take steps towards illustrating the relationship between these two levels of personality in a way that provides insight into pathological dynamics (Pincus et al., 2014). 
Specifically, using the IPC as the structural framework, I investigate how potential mismatches or misalignment in interpersonal efficacies and values relate to interpersonal problems. To do so, I use response surface analysis (RSA; Edwards, 1994; Edwards \& Parry, 1993) to examine how scales developed for the IPC to assess efficacies (CSIE; Locke \& Sadler, 2007), values (CSIV; Locke, 2000), and problems (IIP; Alden, Wiggins, \& Pincus, 1990) are related to each other. This technique avoids issues that arise with more traditional techniques designed to interrogate construct mismatches, such as difference scores, which can collapse information to the point of error. RSA provides more complete information, allowing for the direct comparison of various types of (in)congruence along the entire spectrum of the variables in question. Such analytic strategies have been applied in other areas, such as dyadic relationships (Barranti, Carlson \& Cote, 2017; Schonbrodt, Humberg, \& Nestler 2018). For the current study, I engage RSA for the comparison of interpersonal problems experienced by people who have alignment between their perceived efficacies and values in dominance or warmth compared to various interpersonal problems experienced by combinations of misalignments. Several additional analyses are also included to provide a more complete picture of these dynamics. Following from this theory as well as an analysis using an exploratory sample, I developed and pre-registered the hypotheses that 1) values and efficacies would align for the majority of people in both dimensions and 2) that in both dimensions, mismatches in values and efficacy will predict higher overall reported interpersonal problems (higher elevation on the Inventory of Interpersonal Problems).

This investigation aims to provide a clearer, quantitative description of the relationship between a person's perceived efficacies, values and problems in the critical domain of 
interpersonal functioning. Clarifying this relationship will allow for a connection between personality dynamics and maladaptive interpersonal behavior, serving as a springboard for further research regarding what implications these results have on personality motivation and action. By clarifying our conceptualizations of interpersonal problems, this can ultimately inform clinical processes.

\subsection{Methods}

\subsection{Participants}

The present study leveraged two samples. The first, an undergraduate sample, was used in an exploratory fashion to develop hypotheses which we then submitted for preregistration in the second sample. The second was a community sample. To participate in the study participants had to be 18 years or older at the time of participation. Participants then completed a series of self-report measures based on the IPC.

\subsubsection{Undergraduate sample}

The first sample included a total of 1453 undergraduate students from a large state university. Individuals completed study procedures in exchange for course credit and were consented prior to beginning study protocol. Participants consisted of slightly more females than males $(52.2 \%$ Female, $n=750)$ with an age range of $18-56(M=19 ; S D=1.6)$. The sample was mostly white $(83.8 \%, \mathrm{n}=1217$; Black $=6.1 \%, \mathrm{n}=89 ;$ Asian $=6.7 \%, \mathrm{n}=97$; Other $=1.3 \%, \mathrm{n}=$ 18) with 32 individuals declining to answer this question. 


\subsubsection{Community Sample}

The second sample consisted of 1099 participants recruited through Amazon Mechanical Turk. This sample consisted of slightly more men $(56.3 \%$, Male $=620)$ with two participants identifying their gender as "Other" and an age range of $18-71(M=34.98, \mathrm{SD}=10.37)$ The racial breakdown was more diverse in this sample (White/European $=65 \%, \mathrm{n}=717$; Black $=$ $7 \%, \mathrm{n}=78 ;$ Asian $=16 \%, \mathrm{n}=175 ;$ hispanic $=9 \% ; \mathrm{n}=98 ;$ Multi-Racial $=3 \% ; \mathrm{n}=30$ ).

\subsection{Materials}

\subsubsection{Circumplex Scales of Interpersonal Values (CSIV; Locke, 2000).}

Participants interpersonal values were assessed using the CSIV, a 64-item measure that assesses an individual's motives or values in the context of interpersonal relationships. Participants rated the degree to which they agreed with a range of statements, for example "When I am with him/her/them, it is $[0,1,2,3,4]$ that they show me respect." with 0 being "Not at all' to 4, 'Extremely.'

\subsubsection{Circumplex Scales of Interpersonal Efficacies (CSIE; Locke \& Sadler, 2007)}

Perception of interpersonal efficacies were measured using the CSIE, a 32-item assessment that measures a person's confidence in their ability to successfully achieve certain results in interactions with other people. Participants rated the degree to which they agreed with a range of statements, for example "When I am with others, I can keep the upper hand" on an 11-point Likert Scale, 1 being "Not at all" to 11, "Extremely." 


\subsubsection{Inventory of Interpersonal Problems (IIP; Horowitz, Alden, \& Wiggins, 2000)}

The IIP is a 64-item measure that assesses a person's self- reported difficulties in various interpersonal areas. Participants rate the degree to which they agreed with a range of statements, for example "I fight with other people too much" on a 5-point Likert Scale, 1 being "Not at all" to 5, "Extremely."

\subsection{Data Analytic Plan}

RSA is an implementation of multiple polynomial regression that models the relationship between two predictor variables, their interaction, and an outcome, providing information on what kinds of matches and mismatches between the predictors are more or less associated with

the outcome. It produces four coefficients (a1-a4) that are relevant and interpretable with regards to our study design. We will not be discussing the a5 coefficient as it is more pertinent to dyadic studies looking for prototypical similarity patterns (for example, marital conflict with $\mathrm{X}$ being the one partner's score on conscientiousness and Y being the other partner's score) (Schonbrodt, Humberg, \& Nestler, 2018). The four coefficients describe the shape of the surface above two imaginary axes dividing the XY plane (the horizontal plane; Figures 2-4) of the three-dimensional RSA plot called the line of congruence (relevant to a1, a2) and the line of incongruence (relevant to a3, a4). The line of congruence (LOC), indicates an imaginary line in this plane of the three-dimensional RSA plot corresponding to $\mathrm{X}=\mathrm{Y}$ (in this case, values $=$ efficacies). In other words, the LOC is where the surface above it describes matching $\mathrm{X}$ and $\mathrm{Y}$ values. The line of incongruence (LOIC) is the mismatch analog; it describes a line in the XY 
plane where $\mathrm{X}=-\mathrm{Y}$, the surface above it describes exactly mismatched $\mathrm{XY}$ values (values $>$ efficacies or vice versa).

We will be referring to the RSA coefficients by the titles in the following descriptions, given in italics. A1 and a2 describe what happens to the surface above the LOC; in other words describe what happens to the outcome (i.e., overall problems) when predictors are equal (i.e. values in warmth and efficacies in warmth) A1 (matched slope constant) refers to the slope constant parameter on the surface above the line of congruence. This means that a1 describes the slope above the LOC at the intersection of the LOC and LOIC (which in the absence of curvilinear effect, is the overall slope above that surface). A2 (matched extremity effect) refers to a tendency for values that are further from the mean to either have an increased or decreased value in comparison to that which would be predicted by the general linear trend-- in other words, a curvilinear effect in the LOC.

In contrast, a3 and a4 describe the surface above the LOIC; in other words how an outcome (i.e., overall problems) varies as predictors diverge (e.g. values in warmth increase and efficacies in warmth decrease). A3 (mismatched slope constant) is therefore analogous to a1 and a4 (mismatched extremity effect) is analogous to a2, but in the LOIC. More concretely, this means that as a3 increases or decreases, it reflects how specific mismatches predict an outcome. For example, a3 can tell us that values being greater than efficacies is more predictive of problems or vice versa (as it describes the slope of the surface along the LOIC at the intersection of the LOC and LOIC). Finally a4 (mismatched extremity effect) speaks to the overall effect of mismatching as it represents mismatches that are more extreme in both directions (values more 
than efficacies or efficacies more than values) as having an increased or decreased value in comparison to that which would be predicted by the general linear trend.

As for comparing our two samples the results fall into one of three categories. The first possibility is that the results replicate completely across the two samples. Our criteria for complete replication was that the coefficient replicates quantitatively, such that the coefficient resulting in the community model falls into the confidence interval given by the undergraduate model, and they have the same interpretation (e.g., significant and positive in both samples). The second possibility is that results replicate quantitatively with different interpretation. In our sole instance of this, these were results where a result was significant in one sample and not the other rather than a complete direction reversal. The third possibility is that there is no quantitative replication but the results across the two samples have the same interpretation and therefore are an interpretive replication. In these cases the coefficient falls outside the confidence interval given by the original analyses, but has the same interpretation (e.g., were both positive and significant). Finally, there is the possibility is complete non-replication, such that there is neither quantiative nor interpretive replication. These would be instances in which coefficients in the replication do not fall within the confidence interval given by the initial study, and indicate a different interpretation (e.g., significant in one sample and no-significant in the other, or both significant but with different signs).

For our primary analyses, we were interested in the relationship between dimension specific predictors (values and efficacies within the interpersonal dimensions of dominance or warmth) and overall problems. Therefore we calculated the vector sum scores of each participant on the CSIV and CSIE and then collapsed them into the subscales of dominance and 
warmth. These subscales of the CSIV and the CSIE were used as independent variables with total interpersonal distress scores, as measured by elevation in IIP (average score across all octants of the circumplex) as the independent variable. This produced two three-dimensional RSA plots in each sample for the primary analysis-- one for dominance and one for warmth (Figure 2).

We also conducted several other analyses for the purpose of further elucidating the relationship(s) between levels of functioning (Values, Efficacies, and Problems) and our interpersonal dimensions (Dominance and Warmth). Beyond the primary analyses (Figure 1), I examined (1) Problems with Warmth/Dominance predicted by Values in Warmth/Dominance and Efficacies in Warmth/Dominance, (2) general problems predicted by general warmth and general efficacies, and then (3) the inverse of our primary model, dimension specific outcomes and general predictors.

\subsection{Results}

\subsection{Primary Analyses}

\subsubsection{Effects with quantitative and interpretive replication:}

Within the dominance-based version of this model (dominance-specific values and dominance-specific efficacies predicting overall problems), both the matched extremity effect (a2) and mismatched extremity effect (a4) replicated and were positive, indicating that more extreme matches and more extreme mismatches in dominance values and dominance efficacies were both associated with overall problems in both samples (Figure 2, panels $3 \& 4$ ). 


\subsubsection{Effects with quantitative but not interpretive replication:}

In the warmth analog of this model the mismatched extremity effect (a4) was positive, indicating that more extreme mismatches between values and efficacies in warmth, in either direction, were more predictive of overall problems than the general relationship between values and efficacies would suggest (Figure 2, panels $1 \& 2$ ). This effect, however only reached thresholds of significance in the community sample.

\subsubsection{Effects with interpretive but not quantitative replication:}

Also in the warmth-based models (Figure 2, panels $1 \& 2$ ) both the matched slope constant (a1) and matched extremity effect (a2) had the same interpretations across samples. The matched slope constant was negative, suggesting that in general, as warmth-based values and warmth-based efficacies increase, reported overall interpersonal problems decrease. This result is moderated by the convex matched extremity effect which suggests that, despite this trend, more extreme values in both directions indicate being higher in interpersonal problems than would be predicted by the matched slope constant independently. This effect was greater in magnitude, however in the community sample.

As for the dominance variant of the model (Figure 2, panels $3 \& 4$ ), the matched slope constant was negative in both samples, indicating that as with warmth, in dominance-based values and efficacies increase, reported interpersonal problems decrease. This effect was greater in magnitude in the student sample. 


\subsubsection{Effects that did not replicate:}

The mismatched slope constant (a3) proved to be an unreliable effect in both warmth-based and dominance-based models. With respect to warmth, effect sizes in both samples were small and results were nonsignificant. Therefore, we did not find a relationship between a specific type of mismatch between values in warmth and efficacies in warmth and overall interpersonal problems.

Within the dominance model there was a significant mismatched slope constant in the undergraduate sample, indicating that being high in dominance efficacies and low in dominance values was associated with higher overall interpersonal problems than the reverse. This effect, however, did not appear in the community sample.

\subsection{Exploratory analysis: Within-dimension problems as an outcome (Figure 3, panels 1-4):}

For these models we used warmth/dominance-specific values and warmth/dominance-specific efficacies to predict warmth/dominance-specific problems. It is important to note that unlike the elevation scores (which can be conceptualized as a unidirectional scale of no problems to high problems) these outcome measures are bidirectional in that low scores in warmth problems/dominance problems are indicative of problems with coldness/submissiveness. The zero mark can be interpreted as no problems within that dimension. 


\subsubsection{Effects with quantitative and interpretive replication:}

In the warmth-only model (Figure 3, panels $1 \& 2$ ), matched extremity effects (a2), and mismatched slope constants(a3) replicated across samples. In contrast to the primary analyses with overall problems as the outcome, in these models the matched extremity effect indicated that more extreme values and efficacies in the dimension of warmth, predicted less reported warmth-specific problems. Also in contrast to the primary analyses there was a reliable mismatched slope constant, signifying that efficacies being higher than values in warmth was more predictive of reported problems in warmth, or being higher in values than efficacies predicted problem in coldness (the low pole of the warmth dimension).

With regards to the dominance-specific model (Figure 3, panels $3 \& 4$ ). only the mismatched extremity effect replicated quantitatively, indicating, as with the warmth model and also in contrast to the primary analyses, that more extreme mismatches in dominance were less indicative of problems in dominance (or possibly, more indicative of problems in submissiveness) than the mismatched slope constant would suggest.

\subsubsection{Effects with quantitative but not interpretive replication:}

There was a reversed mismatched extremity effect with respect to the primary analyses-in this model more extreme mismatches in warmth were less indicative of problems in warmth than the mismatched slope constant would suggest, or more indicative of problems in coldness within the undergraduate sample. In the community sample this effect was not significant. 


\subsubsection{Effects with interpretive but not quantitative replication:}

For the warmth-specific model, the matched slope constant was significant and positive in both samples, suggesting that as warmth-values and warmth-efficacies increase, reported warmth-problems also increase. This effect was greater in the student sample.

In the case of the dominance-specific model, matched slope constant, matched extremity effect, and mismatched slope constant has the same interpretation across samples. The results of these were notably similar to the results in the warmth-specific model. In this model the matched slope constant was significant and positive in both samples, suggesting that as dominance-values and dominance-efficacies increase, reported dominance-problems also increase. Additionally the matched extremity effect, while only reaching trendline significance in both samples indicated that more extreme values and efficacies in the dimension of dominance, predicted less reported dominance-specific problems. The mismatched slope constant, reflects that efficacies being higher than values in dominance was more predictive of reported problems in dominance.

\subsubsection{Effects that did not replicate:}

In these models all effects either replicated or had the same interpretation across samples

\subsection{Exploratory analysis: general values and efficacies as predictors (Figure 4, Panels 1-6):}

In these models we used overall values and overall efficacies to predict overall problems, problems in warmth, and problems in dominance respectively. 


\subsubsection{Effects with quantitative and interpretive replication:}

In the outcome where all variables were elevation-scored (Figure 4, panels $1 \& 2$ ), matched slope constant (a1), mismatched slope constant (a3), and mismatched extremity effect (a4) all replicated. For the matched slope constant, there was a small effect size, only significant in the community sample indicating that overall values and overall efficacies were possibly slightly associated with overall problems. The mismatched slope constant in both models showed a strong effect indicating that higher overall values and lower overall efficacies were more predictive of overall problems. Finally the mismatched extremity effect, moderately strong, significant, and positive across samples showed that, like the primary analyses extremity of mismatch was more indicative of overall problems.

In the model overall problems and values predicting warmth specific problems, matched slope constant, matched extremity effect, and mismatched extremity effect all replicated. However for all three measures effect sizes were small with unreliable significance. A slight positive matched slope constant was found to be significant in only the student sample. The matched extremity effect reached trendline significance in the student sample and was not significant in the community sample and the mismatched extremity effect was only significant in the community sample. However these effects indicated slight tendencies towards warmth-specific problems being indicated by increased overall values and increased overall efficacies, with the more extreme matches and mismatches being less indicative of warmth problems than the linear trends would suggest.

Finally in the dominance outcome, overall values and efficacies model (Figure 3, panels $5 \&$ 6), matched slope constant and matched extremity effect replicated across samples. In this 
case increased overall values and increased overall efficacies predicted increased dominance problems, with more extreme values being more indicative of problems in dominance than predicted by the linear trend.

\subsubsection{Effects with quantitative but not interpretive replication:}

There were no instances of this in this set of analyses.

\subsubsection{Effects with interpretive but not quantitative replication:}

The only effect in these models that did not replicate but had the same interpretation was the mismatched extremity effect for dominance. In both samples this effect was moderately-sized negative and significant indicating that overall efficacies being higher than overall values was more predictive of dominance-specific problems.

\subsubsection{Effects that did not replicate:}

In the elevation-only model the coefficient representing matched extremity effect was negative and significant in the undergraduate sample, however was positive and insignificant in the community sample, with effect sizes being relatively small in both cases. Additionally in the model with warmth-specific problems as an outcome, the mismatched slope constant, while nonsignificant and positive in the community sample was significant and negative in the community sample, also with small effect sizes.

\subsection{Discussion}

This study sought to add to the body of literature focusing on personality processes by investigating the associations between interpersonal problems and intrapersonal misalignment, 
specifically the personality levels of interpersonal values and efficacies. In general mismatching was predictive of interpersonal problems, which aligns with predictions regarding the negative impact of intrapersonal conflict upon a person's well being (Higgins, 1987; Leary, 1957). Furthermore, our choice to use RSA provided us with results which suggest more specific relationships between types of mismatches and types of outcomes, within the broader mismatch-problems relationship. This both quantifies and confirms older theories and intuitions, and expands upon them, providing a basis for new directions in investigating these personality mechanisms.

From our primary analyses (and also in our all-elevation model), I found that when using overall problems as an outcome, larger mismatches in either direction resulted in higher predicted outcomes (mismatched extremity effect). This is in line with our predictions, as well as the theory driving this investigation, suggesting that within-person level misalignment has a negative impact upon interpersonal functioning. Additionally there was a negative matched slope constant. Because these predictors exist on bipolar scales (warmth/dominance versus coldness/submissiveness respectively), this could mean that higher values and efficacies in warmth/dominance were negatively associated with overall interpersonal problems or that higher values and efficacies in the opposing pole of coldness/submissiveness were positively associated with overall interpersonal problems (Figure 3, Panels 1-4; Figure 4, Panels 1 \& 2). Additionally, in our primary analyses, there were positive matched extremity effects for both warmth and dominance-- in other words, as scores on warmth values and efficacies moved further from the average, they became more positively associated with interpersonal problems, despite the overall 
negative relationship between interpersonal problems and higher matching. These effects may provide evidence for maladaptivity as a consequence of extremity.

Finally, these primary analyses suggest that having any mismatch in values and efficacies (whether values are greater than efficacies or efficacies are greater than values) was predictive of greater overall interpersonal problems (no reliable mismatched slope constant, but significant positive mismatched extremity effect). This result alone is in contrast to predictions that may be derived from theory regarding the relationship between values and efficacies, which emphasizes problems caused by efficacies being lower than values (Azjen \& Fishbein, 1987).

However, our exploratory models suggest that within this nonexistent mismatched slope constant in our primary analyses there are possibly multiple mechanisms collapsed into the elevation score (calculated by averaging the IIP score across octants). Firstly, the similarity between the outcomes across dominance and warmth in our exploratory dimension-specific models suggests that these models provide us with information with regard to how dimension-specific values, efficacies and problems relate, as opposed to how these interpersonal levels relate to dominance or warmth specifically. They also suggest that, when constrained to a specific dimension, there are effects of specific mismatches on predicting specific types of problems. With respect to these dimension-specific results, because of the way the mismatched slope constant is calculated, the presence of a negative mismatched slope constant could mean two things, as with the matched slope constant of the primary analysis. The first explanation is that a mismatch of values being greater than efficacies could be associated with problems with the low pole of that dimension (coldness for warmth and submissiveness for dominance). The second is that a negative mismatched slope constant could be a function of efficacies exceeding 
values predicting problems, with the high pole of the dimension (warmth for warmth and dominance for dominance).

In contrast to the models from the primary analyses, the two possible contributors to the effect can be disentangled. This is because the outcome variable (IIP) is also bipolar, so the predicted model shape for each explanation are different. Take the hypothetical participants, Liza and Ivan within the dimension of warmth. Ivan values warmth (score of +1.0, CSIV_LOV), but has low self-ratings of efficacy (-2.0, CSIE_LOV). Liza, on the other hand, feels efficacious in warmth (+2.0, CSIE_LOV) but has low self-ratings of values in warmth (-1.0, CSIV_LOV). If explanation 1 were true, that values greater than efficacies predict problems with coldness, we would expect Ivan to have a negative score, and Liza to have a score close to zero. However if explanation 2 were true, that efficacies greater than values predict problems with warmth, we would expect Liza to have a positive score, and Ivan to have a score close to zero. If we place Ivan and Liza onto the actual plot given by the models (Figure 3, panels 1\&2), Ivan ends up having a relatively high score of problems with coldness $(-2.0$, IIP_LOV). Liza ends up indicating slight problems with warmth $(0.005$, IIP_LOV). This would align with explanation 1 , that the mismatched slope constant is being driven by people like Ivan, who have problems with coldness, and a values-greater-than-efficacies misalignment, and not people like Liza who have close to an absence of problems in the warmth dimension and an efficacies-greater-than-values misalignment.

Explanation 1 is also reflected in the directionality of the mismatched extremity effect, which was significant and negative in the student sample, and replicated quantitatively in the community sample, although it was not significant. Our primary analyses and our other 
exploratory analyses suggest that extremity produces more problems. Therefore the direction of this effect should align with the IIP pole driving the shape of the model. In the student sample, more extreme scores become more negative, supporting the explanation that values-greater than efficacies predict problems with coldness. If the mismatched slope constant was driven by the Lizas of the world, with efficacies-greater-than-values mismatching predicting problems with warmth, extremity would then be less problematic as Liza 2.0, who has a more extreme mismatch than Liza (+2, CSIE_LOV; -2; CSIV_LOV) also has essentially no reported problems in the warmth dimension, and Liza $3.0(+2$, CSIE_LOV; -3, CSIV_LOV) begins to have problems with coldness. It seems much more likely that the effects of extremity that we see in this model are being driven by the Ivans, with a values-greater-than efficacies mismatch, as we would see the respective Ivan 2.0 and 3.0 having increasingly more extreme problems with coldness. Finally, the first interpretation, that these graphs demonstrate problems with the low-pole of the dimension and an efficacies-less-than-values discrepancy, also agrees with findings from attitudinal-behavioral theory, in that, despite valuing the positive pole of the dimension (such as tendencies towards warmth and warmth), people with lower efficacies in that dimension, engage in behaviors in the opposing pole of that dimension (tendencies towards separation and disaffiliation), even though they find them problematic. This effect persisting across both the dimensions of dominance and warmth suggest that this mechanism is not specific to either of these IPC dimensions, and is instead a general tendency in dimension-restricted models.

Additionally, problems within a dimension were associated with higher values and efficacies in that dimension (matched slope constant). This may be due to people scoring higher 
in values and efficacies in a dimension simply engage more with that dimension and therefore experience more problems in it. The fact that people who have the values-greater-than-efficacy mismatch experience, in general, problems with coldness, while people who are high in both experience problems with warmth, suggest that there are indeed content-based differences in the interpersonal problems that different personality subgroups experience.

Our third set of exploratory analyses suggests that, when looking at general levels of values and efficacies (not using the dominance/warmth subscales at all), values being greater than efficacies is most predictive of perceived interpersonal problems (Figure 4, panels $1 \& 2$ ). The effect size of this finding was strong in both samples and replicated well. However there are other factors worthy of consideration that could be contributing to the strength of this effect. For example, it is unclear what "overall values" measures-- high "overall values" may be a sign of identity diffusion, which would be associated with interpersonal problems for a variety of reasons beyond misalignment between values and efficacies specifically. In contrast it seems reasonable to link overall interpersonal efficacies to a general sense of self-efficacy. Therefore, while having high matched values and efficacies is only slightly associated with problems rendering the discrepancy between the two levels central to this particular model, this result may not fully generalize outside of this ambiguous "overall values" measure.

Finally, while for most of our analyses the dimensions of dominance and warmth behaved similarly to each other, the exception to this is in our last set of exploratory analyses (Figure 4, panels 3-6). In these models we see virtually no consistent effect for warmth, while we see moderately strong effects replicate for dominance in matched slope constant, matched extremity and mismatched slope constant. As with the other models using IIP-dominance as an 
outcome, problems with the pole of the dominance scale corresponding to dominance land in the efficacies higher than values while problems with the opposing pole (submissiveness) land with people whose values are higher than their efficacies (broadly speaking). This is suggestive of a relationship between trait dominance variation and the levels of interpersonal values and efficacies that people express.

\subsection{Strengths and Limitations}

While this study had many strengths there were a few limitations. Our manipulation of the IPC vector variables may have eliminated valuable information. Implementing RSA within the IPC space involves uncoupling dominance and warmth vector components, which is not ideal. This may eliminate valuable information contained in the data; for example, one would not expect people who are dominant and cold to have the same interpersonal experience as those who are dominant and warm. However, these specifics are tangential to the broader question regarding the effect of misalignment within these dimensions. Additionally, the outcome variable for our primary analyses, IIP elevation, reflects general interpersonal difficulty, encompassing all varieties of distress. Therefore, even though the problems experienced may be different, the prediction is that misalignment will predict problems-- an effect that this measurement accounts for. However our inclusion of the exploratory analyses attempted to address this issue to some degree, and our application of RSA provides us with much more information than could be achieved using other means, such as difference scores. While a logical future direction would be to examine the dimensions of dominance and warmth in conjunction, we believe that for the current study this choice is justified as this design still answers our basic questions, this RSA package in $\mathrm{R}$ is already established, and the three-dimensional nature of the current design 
produces useful visualizations and more intuitive, yet still valid, interpretations. In addition, our results suggest that extremity could in some cases impact effects (significant matched extremity effects), which could have implications with regards to the clinical generalizability. However personality difficulties are distributed throughout the general population and there is little reason to believe that personality mechanisms change completely once they reach a magic threshold.

With regard to its representation of the general population, this study had the strength that we were able to use an undergraduate and community sample from different sources with a total of 2,554 participants. Therefore results that these two samples share are likely to generalize to the population. In addition this statistical method is relatively advanced and provided us with much more textured results than could have been achieved without it.

In sum, this study elaborates upon the relationship between the levels of values and efficacies, producing results that both align with previous work across a variety of theoretical perspectives, and enriching the conceptualization of this relationship via the complexity allowed by RSA. It also establishes the IPC as a platform for comparing within-person processes and RSA as a technique that produces more fine-grained and nuanced results for this type of inquiry than traditional methods of assessessing alignment. 


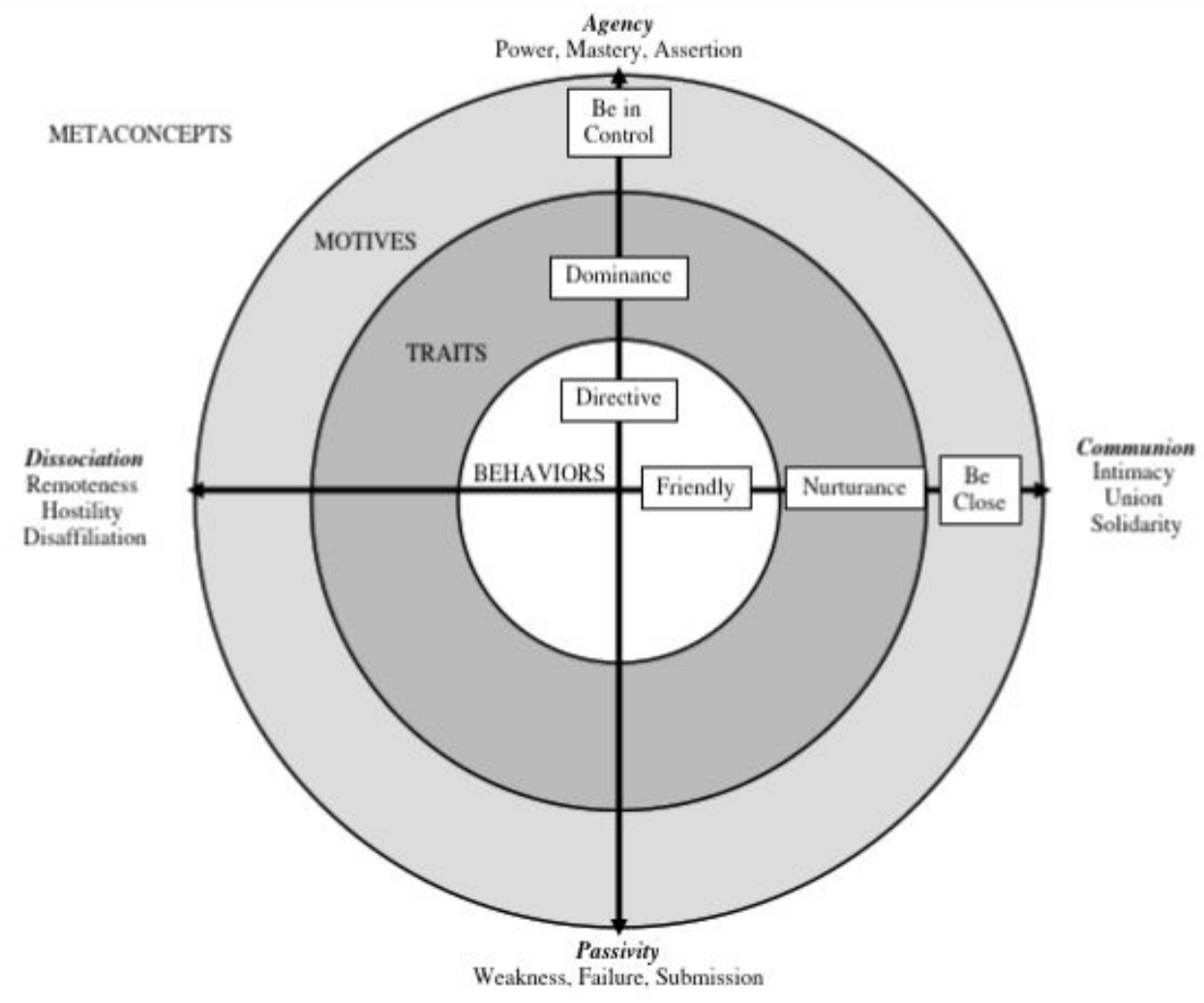

Figure 1

Interpersonal circumplex 

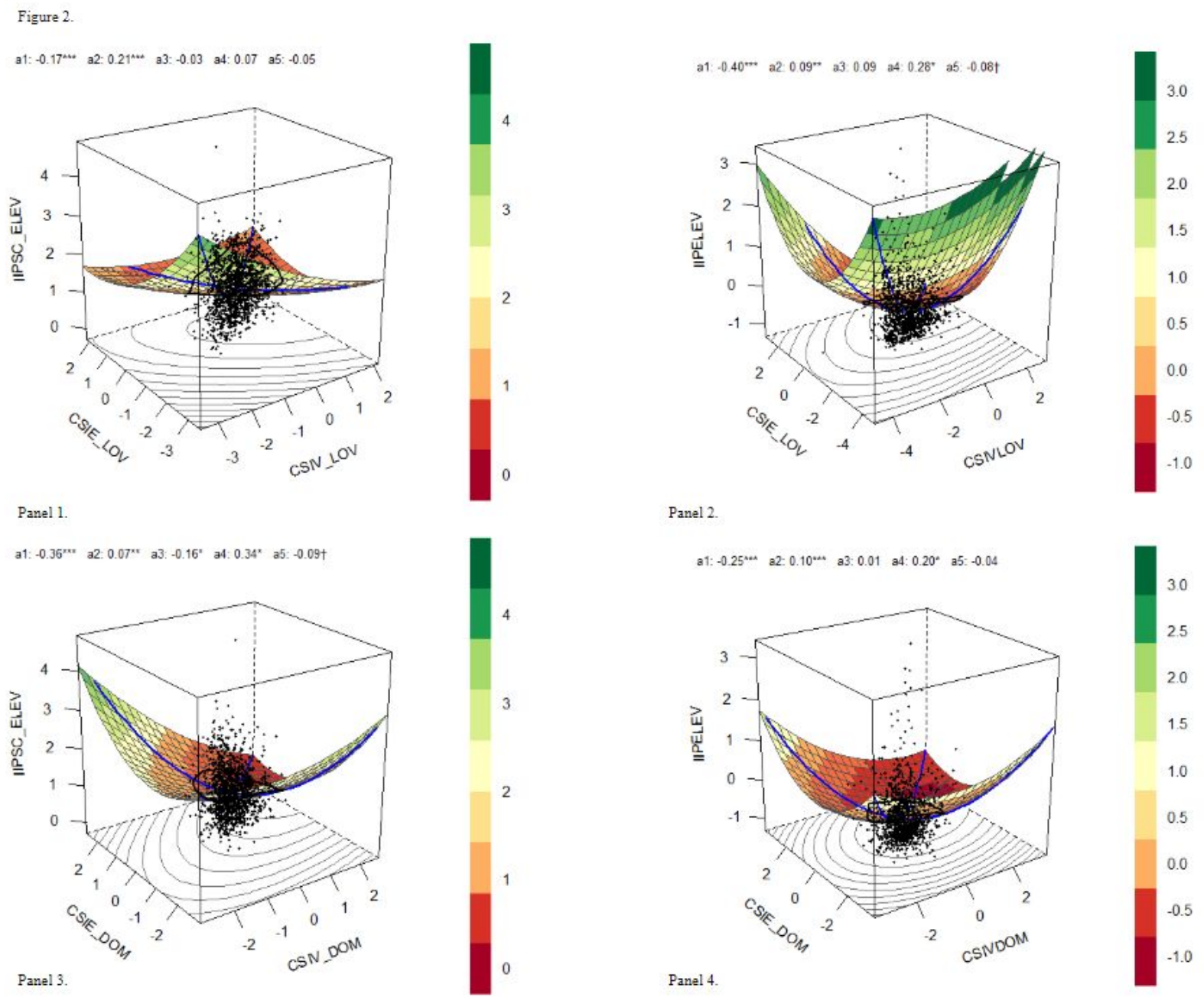

Figure 2. 
Figure 3.
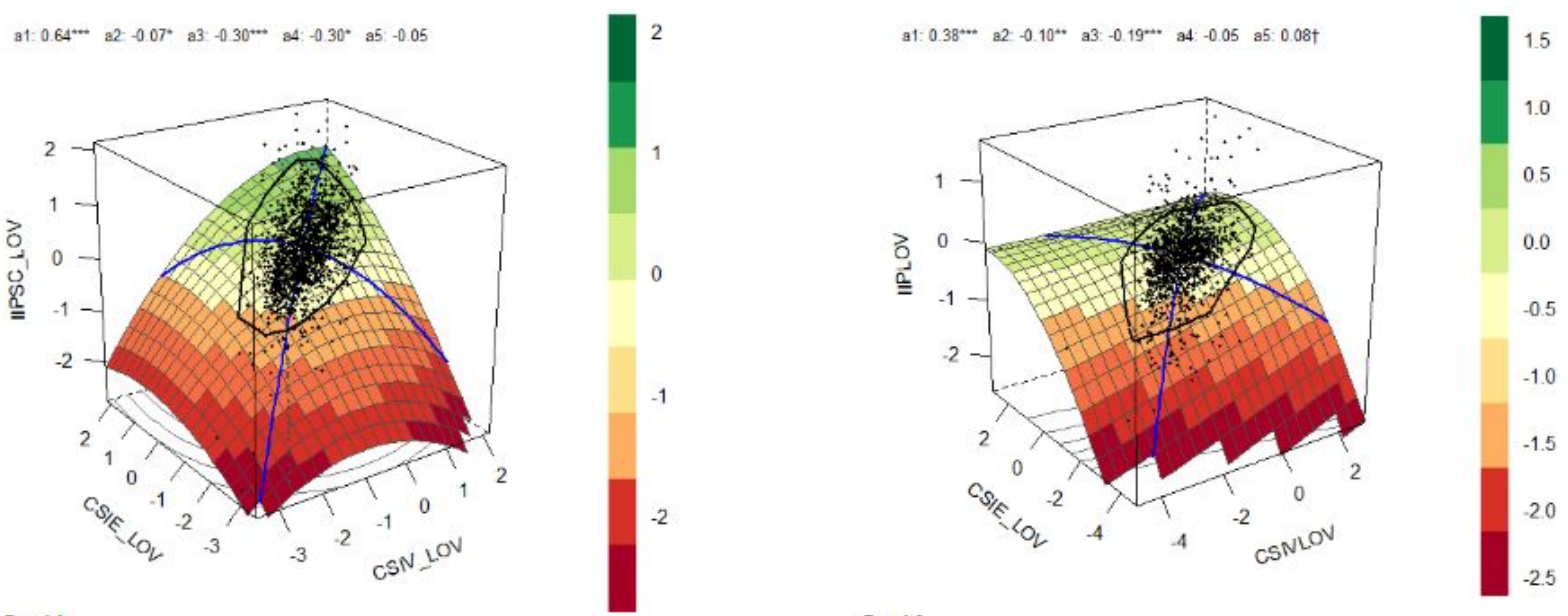

Panel 1.
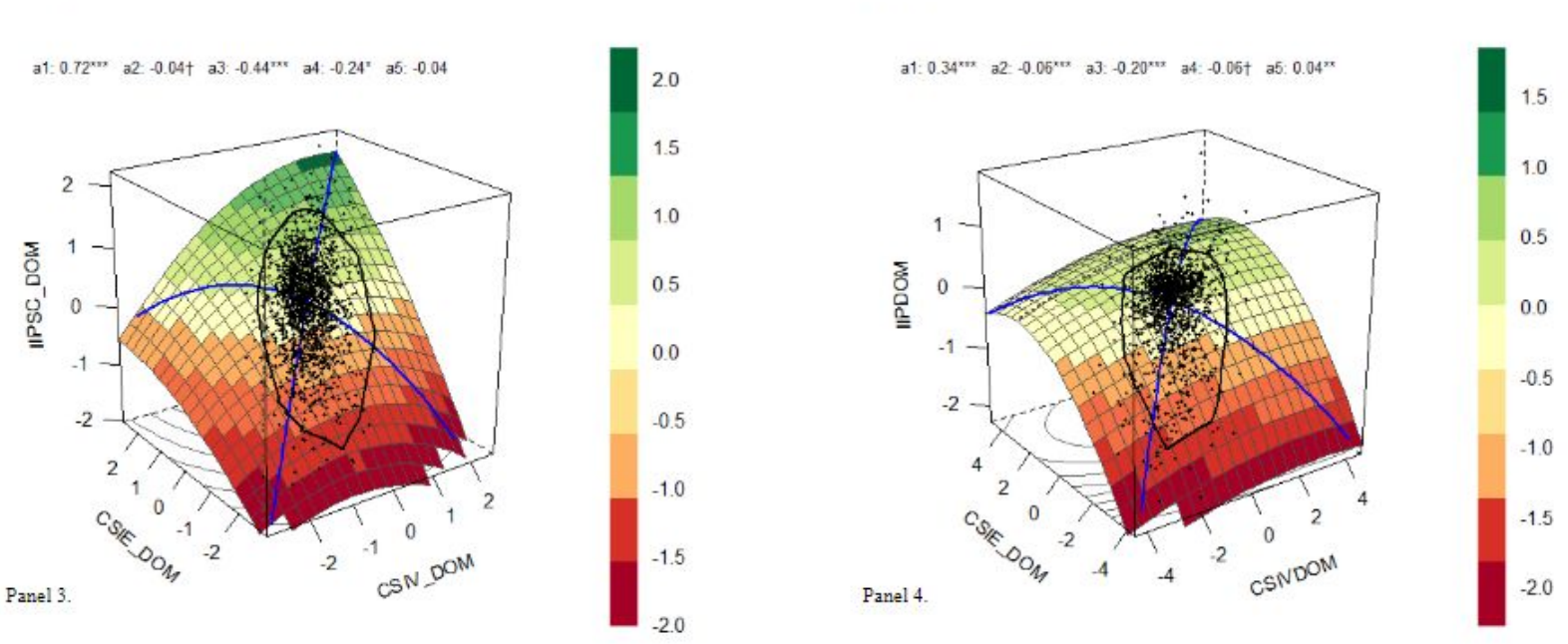

Figure 3. 

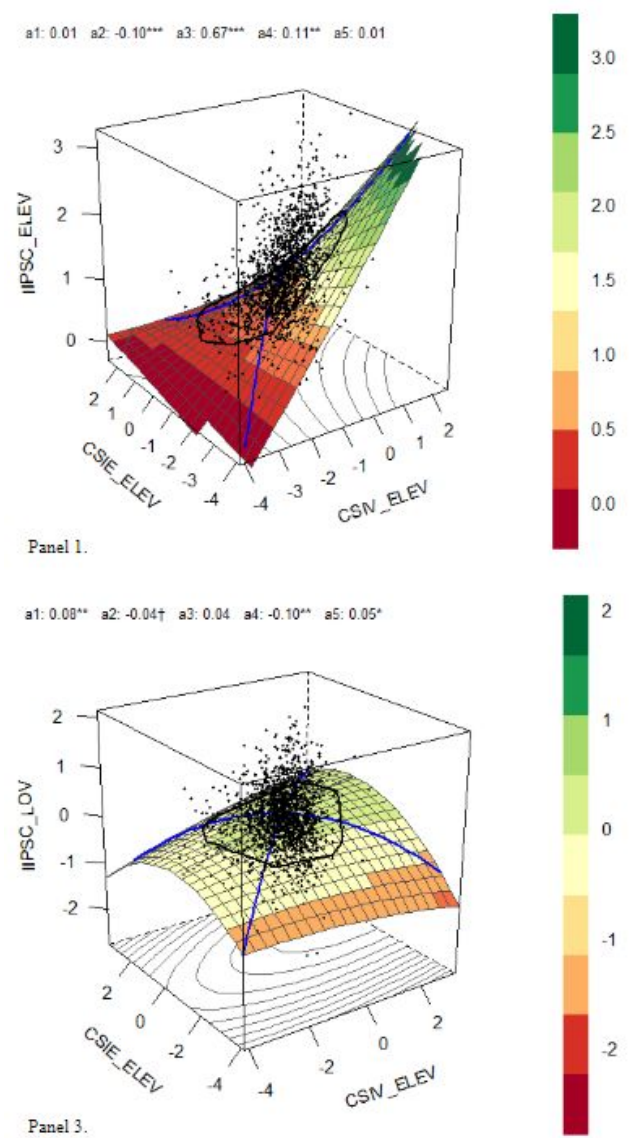

a1: $0.22^{\text {nst }}$ a2: $0.09^{+\cdots}$ a3: $-0.09^{\circ+}$ a4: 0.06 a5: -0.04

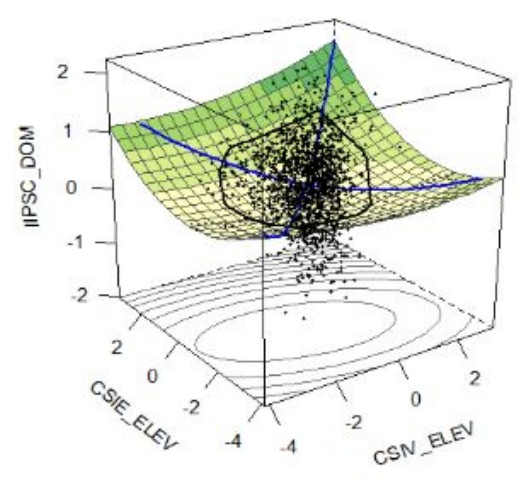

Panel 5.
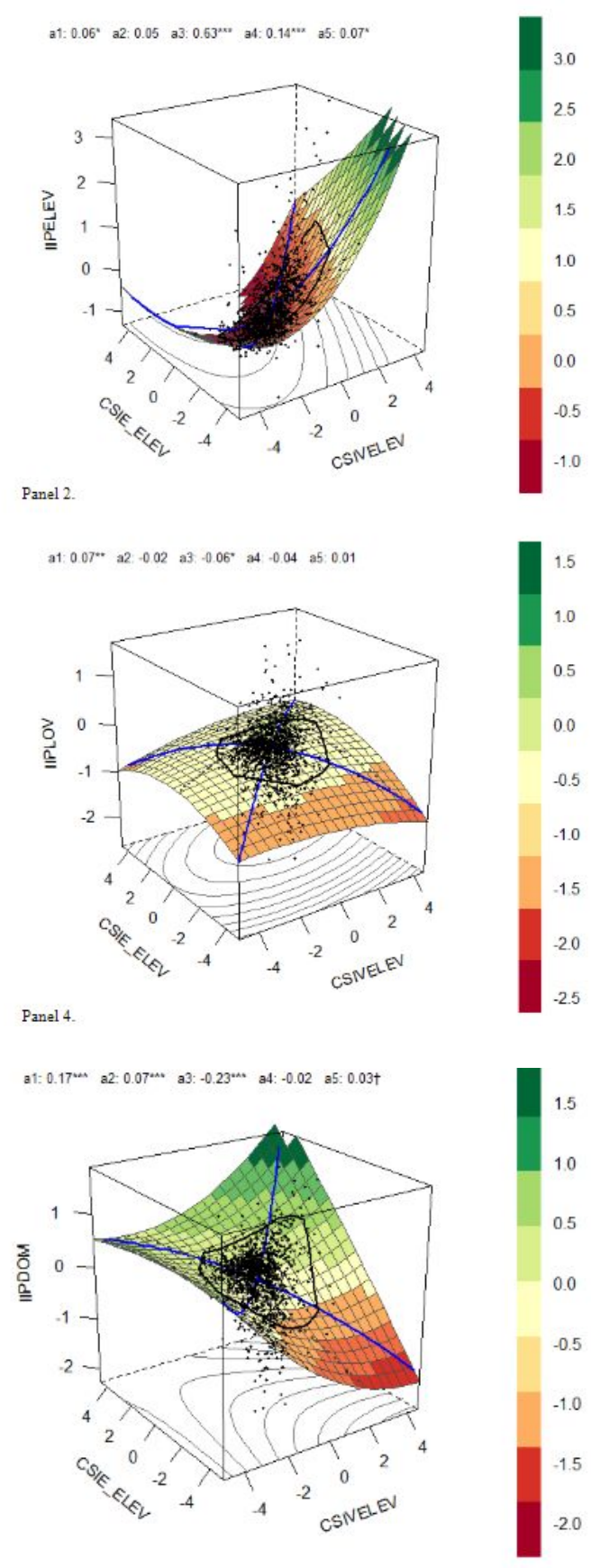

Panel 6.

Figure 4. 


\section{Bibliography}

Ajzen, I., \& Fishbein, M. (1977). Attitude-behavior relations: A theoretical analysis and review of empirical research. Psychological Bulletin, 84, 888-918.

Baldwin, M. W. (1992). Relational schemas and the processing of social information. Psychological Bulletin, 112, 461-484.

Bandura, A. (1977). Self-efficacy: Toward a unifying theory of behavioral change. Psychological Review, 84(2), 191-215.

Barranti, M., Carlson, E. N., \& Côté, S. (2017). How to test questions about similarity in personality and social psychology research: Description and empirical demonstration of response surface analysis. Social Psychological and Personality Science, 8(4), 465-475.

Clark, L.A. (2005) Temperament as a unifying basis for personality and psychopathology. Journal of Abnormal Psychology, 114, 505-521.

Conte, H. R., \& Plutchik, R. (1981). A circumplex model for interpersonal personality traits. Journal of Personality and Social Psychology, 40, 701-711.

Edwards, J. R., \& Parry, M. E. (1993). On the use of polynomial regression equations as an alternative to difference scores in organizational research. Academy of Management Journal, 36, 1577-1613.

Edwards, J. R. (1994). Regression analysis as an alternative to difference scores. Journal of Management, 20, 683-689.

Eldredge, K. L., Locke, K. D., \& Horowitz, L. M. (1998). Patterns of interpersonal problems associated with binge eating disorder. International Journal of Eating Disorders, 23(4), 383-389. 
Feather, N. T. (1982). Human values and the prediction of action: An expectancy-valence analysis. In N.T. Feather (Ed.), Expectations and actions: Expectancy-value models in psychology (pp. 263-289).Hillsdale, NJ: Lawrence Erlbaum Associates, Inc.

Foa, U. G. (1961). Convergences in the analysis of the structure of interpersonal behavior. Psychological Review, 68(5), 341-353.

Fournier, M. A., Moskowitz, D. S., \& Zuroff, D. C. (2008). Integrating dispositions, signatures, and the interpersonal domain. Journal of Personality and Social Psychology, 94(3), $531-545$.

Fournier, M. A., Moskowitz, D. S., \& Zuroff, D. C. (2009). The interpersonal signature. Journal of Research in Personality, 43(2), 155-162.

Gurtman, M.B. (1992), Construct Validity of Interpersonal Personality Measures: The interpersonal Circumplex as a Nomological Net. Journal of Personality and Social Psychology 63(1), 105-118.

Higgins, E. T. (1987) Self-Discrepancy: A Theory Relating Self and Affect. Psychological Review 94(3), 319-340.

Hopwood, C.J., Pincus, A.L., \& Wright, A.G.C. (in press). The interpersonal situation: Integrating clinical assessment, formulation, and intervention. In D. Samuel \& D. Lynam (Eds.), Using basic personality research to inform personality pathology. New York, NY: Oxford University Press.

Horowitz, L. M., Alden, L. E., Wiggins, J. S. and Pincus, A. L. 2000. Inventory of Interpersonal Problems: Manual, San Antonio, TX: The Psychological Corporation. 
Horowitz, L. M., Rosenberg, S. E., \& Bartholomew, K. (1993). Interpersonal problems, attachment styles, and outcome in brief dynamic psychotherapy. Journal of Consulting and Clinical Psychology, 61(4), 549-560.

Kiesler, D. J. (1983). The 1982 interpersonal circle: A taxonomy for complementarity in human transactions. Psychological Review, 90(3), 185-214.

Leary, T. (1957). Interpersonal diagnosis of personality: A functional theory and methodology for personality evaluation. New York: Ronald Press Co.

Locke, K.D. (2000) Circumplex Scales of Interpersonal Values: Reliability, Validity, and Applicability to Interpersonal Problems and Personality Disorders. Journal of Personality Assessment, 75(2), 249-267.

Locke, K.D., Sadler, P (2007) Self-efficacy, values, and complementarity in dyadic interactions: Integrating interpersonal and social-cognitive theory. Personality and Social Psychology Bulletin, 33(1), 94-109.

Locke, K. D. (2015). Agentic and communal social motives. Social and Personality Psychology Compass, 9(10), 525-538.

Lukowitsky, M.R., Pincus, A. L. (2011)The pantheoretical nature of mental representations and their ability to predict interpersonal adjustment in a nonclinical sample. Psychoanalytic Psychology, 28(1), 48-74.

Luyten, P., \& Blatt, S. J. (2013). Interpersonal relatedness and self-definition in normal and disrupted personality development: Retrospect and prospect. American Psychologist, 68(3), 172-183. 
Mischel, W., \& Shoda, Y. (1998). Reconciling processing dynamics and personality dispositions. Annual Review of Psychology, 49(1), 229-258

Pincus, A. L., Lukowitsky, M. R., Wright, A. G. C., \& Eichler, W. C. (2009). The interpersonal nexus of persons, situations, and psychopathology. Journal of Research in Personality, 43(2), 264-265.

Pincus, A.L., Ansell, E. B. (2012). Interpersonal Theory of Personality In A. L. Pincus and E. B. Ansell(pp. 141-160) Handbook of psychology, personality and social psychology (2. Aufl. ed.). Somerset: Wiley.

Pincus, A.L., Sadler, P. , Woody E., Roche, M. J., Thomas, K. M., and Wright, A. G. C. (2014) Multimethod Assessment of Interpersonal Dynamics, In: Hopwood C. J., Bornstein R. F., editors. Multimethod Clinical Assessment. New York: Guilford Press; pp. 51-85.

Plomin, R., DeFries, J. C., Knopik, V. S., \& Neiderhiser, J. M. (2016). Top 10 replicated findings from behavioral genetics. Perspectives on Psychological Science, 11(1), 3-23.

Samuel, D. B., \& Widiger, T. A. (2008). A Meta-Analytic Review of the Relationships Between the Five-Factor Model and DSM-IV-TR Personality Disorders: A Facet Level Analysis. Clinical Psychology Review, 28(8), 1326-1342.

Wiggins, J. S. (1979). A psychological taxonomy of trait-descriptive terms: The interpersonal domain. Journal of Personality and Social Psychology, 37(3), 395-412.

Wright, A. G. C., Pincus, A. L., Hopwood, C. J., Thomas, K. M., Markon, K. E., \& Krueger, R. F. (2012). An interpersonal analysis of pathological personality traits in DSM-5. Assessment, 19(3), 263-275. 
